\title{
La variation du français parlé au Gabon : transgression ou progression ?
}

Magali ITALIA, Université de Provence - LPL

\section{Introduction}

Une transgression s'effectue par rapport à une norme prescrite, admise, connue et généralement respectée par tous. D’un point de vue des sciences du langage, la norme d'une langue renforce la cohésion sociale car elle permet à des individus de la communauté linguistique dont elle dépend de se reconnaître entre eux. La transgression de cette norme entraîne un préjugé négatif. Mais par le biais de la variation, la transgression cesse d'être négative car l'appropriation entraine une adéquation entre langue et société, langue et vision du monde. La preuve en est que la langue française a suivi l'évolution de la société française au cours des siècles. Considère-t-on que, parce que nous ne parlons pas le français classique du dix-septième siècle, le français d'aujourd'hui est transgressif ? Les normes pré-établies ne sont-elles pas vouées à évoluer? Une langue, parce qu'elle suit l'évolution de la société qui la pratique, déroge aux règles prescrites, s'en crée de nouvelles et s'adapte aux besoins langagiers de cette même société. Pour éclairer ces propos, nous étudierons le cas du Gabon où le français est l'unique langue officielle. En effet, contrairement à d'autres pays d'Afrique francophone comme le Sénégal ou la Centrafrique, le Gabon ne présente sur son territoire aucune langue vernaculaire d'ampleur assez importante pour devenir la langue véhiculaire entre les différentes ethnies. Le français remplit ce rôle. Il est à l'image de la société métropolitaine qui l'emploie mais non à l'image de la société gabonaise. Pour devenir langue véhiculaire, le français a été contraint de passer par le biais de l'appropriation qui entraine une variation de la langue. La variation de la langue, synonyme de changements linguistiques, doit-elle être perçue comme une transgression par rapport aux normes prescrites ou comme le symbole d'une progression avec adéquation entre société et langue ?

À partir de l'étude de corpus de français parlé au Gabon, nous réfléchirons à la manière dont il faut considérer l'évolution de cette langue étrangère devenue véhiculaire. Doit-elle rester figée dans les normes prescriptives avec le risque que la population gabonaise la rejette ou bien créer de nouvelles normes?

Nous cernerons le rôle du français à travers son statut d'unique langue officielle, de langue véhiculaire puis de vernaculaire. Nous donnerons des exemples de français vernacularisé. La variation touche en premier le lexique puis la structure grammaticale. Nous terminerons par le rejet du terme 'transgression' pour celui moins négatif de 'progression'. 


\section{Situation du français au Gabon}

Pour bien comprendre la situation du français au Gabon, besoin est de prendre en considération plusieurs paramètres : le français en tant que langue officielle, puis comme langue véhiculaire et vernaculaire.

\subsection{Français unique langue officielle}

Le premier paramètre concerne le statut privilégié du français au Gabon. En effet, le français est l'unique langue officielle du Gabon.

Les langues ethniques ne possèdent aucun statut officiel en raison de la faible part de la population parlant chacune d'elle. Aucune d'elle n'apparaît suffisamment développée pour obtenir une reconnaissance et un statut officiel en tant que langue nationale. En l'absence d'une langue ethnique de diffusion nationale, le français apparaît comme la seule langue à pouvoir remplir ce rôle. Le français domine toute autre langue par sa reconnaissance officielle et il est l'unique langue :

- $\quad$ « du pouvoir politique ;

- du pouvoir financier et économique, dans les structures de grande dimension ;

- du pouvoir par l'écrit : système scolaire, presse écrite, correspondance, relations dans l'entreprise ou entre administrations, maîtrise de la technologie moderne » (MoussirouMouyama et de Samie 608);

- de la véhicularité.

Pour marquer son statut élevé, Karine Boucher et Suzanne Lafage comparent l'importance officielle du français «à celle qu'il a dans un pays où il est langue maternelle de la population » (XVIII). Le français, en tant que langue officielle, doit être compris comme le français normé. Françoise Gadet définit la norme comme un «effet de la standardisation qui incite à sacraliser la forme de langue préconisée comme la meilleure façon de parler et surtout d'écrire » (126). Cette norme prescriptive, définie comme étant le modèle à rejoindre, hiérarchise donc les normes de fonctionnement concurrentes, même si elles prennent souvent les apparences des normes descriptives. Cette définition sous-entend que la norme correspond aux règles qui régissent les codes de la langue écrite sans faire de distinction avec le français oral. Les structuralistes ont réduit la norme «à une fausse conscience ou à une idéologie linguistique confondue avec le prisme»; ce qui revient à une «conception de la langue comme un objet homogène, abstrait de ses conditions de production historique et sociale, [accompagné par 1'] exclusion du sujet énonciateur, envisagé comme un locuteur sans identité » (Baggioni cité dans Moreau 218). 
La norme se confond avec le bon usage, tout comme Adeline Larissa Simo Nguemkam-Souop, nous affirmons qu' « on la trouve dans les dictionnaires et les ouvrages de grammaire qui se chargent de légiférer les usages (...). La parole ainsi visée est idéale. Cette perception métalinguistique homogénéisante qui fait de la langue un objet clos est une pure fiction »(60). Parce qu'elle n'est pas pratiquée par la population dans la vie quotidienne, cette norme est devenue une norme fantasmée, c'est-à-dire que les membres de la communauté linguistique se forgent un ensemble de conceptions sur la langue et son fonctionnement social, qui ne présentent parfois qu'une faible zone d'adhérence avec le réel.

Un secteur important des normes fantasmées concerne la manière dont les membres de la communauté conçoivent ce qu'est la norme, la part que prennent les grammairiens et les autres institutions normatives dans sa définition, et les groupes sociaux qui la détiennent. On peut ainsi mettre parfois en évidence, dans certaines communautés - c'est le cas pour la francophonie -, qu'une proportion importante de locuteurs se représentent la norme comme un ensemble abstrait et inaccessible de prescriptions et d'interdits, qu'ils ne voient s'incarner dans l'usage de personne et par rapport auquel tout le monde se trouve donc nécessairement en défaut. (222-223)

Ainsi la norme, dans une perspective de langue parfaite, ne serait que pur fantasme et par cette conception même d'idéal, cette norme ne sera jamais atteinte. Toujours selon Simo Nguemkam-Souop (60), à la suite de Jean-Paul Bronckart (110), cette conception de la norme «adopte un discours classificatoire et hiérarchisant »(110) «qui aboutit à une morale de la langue. Lorsque la norme n'est pas respectée, les jugements portés sur le locuteur en infraction dépassent très souvent le cadre d'une évaluation linguistique et affectent parfois sa personnalité » (126).

La norme telle qu'envisagée ci-dessus n'apparaît que comme une abstraction, une pure fiction, provoquant des préjugés sur ceux qui ne l'emploient pas correctement. Cette conception est peu conforme à la réalité du terrain et exempte de toute considération sociale.

L'organisation des faits linguistiques ne peut être indépendante de la population qui pratique la langue. Les normes influencent obligatoirement la langue que les locuteurs utilisent. La réalité du pays où elle est pratiquée est également un facteur important à prendre en considération. Comme le souligne à juste titre Simo Nguemkam-Souop (63), parce que n'ayant pas par tradition comme en France, la culture (ou le culte) de l'écrit, le français a pu subir des modifications plus ou moins importantes selon les pays, ce qui a permis « une meilleure reconnaissance sociale » de la langue par la population qui la pratique. 


\subsection{Français langue véhiculaire}

Le second paramètre concerne l'appropriation du français par la population du Gabon. Cette appropriation se compose dans un premier temps de la véhicularisation du français puis de sa vernacularisation. Le français approprié est avant tout une langue orale, qui se transmet essentiellement par le biais de l'oralité. Il ne provient donc pas du français normé, ni écrit.En plus de son statut officiel exclusif, le français est devenu le véhiculaire au Gabon. Auguste Moussirou-Mouyama et Thierry de Samie résument la situation ainsi : «le français joue un rôle de langue véhiculaire écrasante, au-delà même de son statut de langue officielle » (608). Le corpus du français se révèle important. Boucher et Lafage expliquent les raisons de ce corpus élevé :

«Scolarisation totale en français, pour la quasi-totalité des jeunes générations (Taux de scolarisation 94\%, Taux de scolarisation au primaire $94 \%$ et au secondaire $35 \%$, taux d'alphabétisation des hommes et des femmes de 15 à 24 ans (99\% et 97\%) les plus élevés d'Afrique Noire - Chiffres de l'Unicef 2007-2010),

- Urbanisation intense qui impose pour l'intercommunication l'usage d'un véhiculaire adapté à la modernité,

- $\quad$ Absence de langue gabonaise à rôle véhiculaire national,

- $\quad$ Présence d'un certain nombre d'étrangers, certes moins nombreux mais souvent plus scolarisés qu'autrefois et plus ouverts à l'utilisation d'une langue de grande communication comme le français qu'à l'apprentissage d'une langue locale à diffusion restreinte,

- Culte de la modernité et désir d'ouverture vers les technologies les plus avancées, pour l'instant surtout accessibles en français » (XX).

Le français apparaît comme la langue «gagne-pain» qui assure la survie de l'individu, langue d'accès au bien-être social (réel ou supposé), langue d'ouverture culturelle accès à la lecture étrangère, notamment française ». De plus, « la langue française lamine les différences entre les nombreuses communautés ethno-linguistiques qui la composent pour s’imposer comme langue véhiculaire » (Moussirou-Mouyama et de Samie 611).

Le phénomène de l'urbanisation importante du pays n'est pas étranger à l'utilisation du français en tant que véhiculaire. L'urbanisation intense du pays $-86 \%$ selon l'Unicef, $80 \%$ selon le site de la présidence du Gabon - brasse toutes les ethnies du Gabon ainsi que les étrangers présents sur son sol, et s'accompagne d'un fort plurilinguisme'. En l'absence d'une langue ethnique autochtone de diffusion nationale, la ville favorise l'expansion $\mathrm{du}$ « français qui profite de la diversité linguistique dans un désert démographique et de l'urbanisation déséquilibrée du Gabon » (Moussirou-Mouyama 49). Selon Louis-Jean Calvet, 
la ville est la quintessence du plurilinguisme, un pays tendra d'autant plus vers l'unification linguistique qu'il y aura un taux d'urbanisation élevé. Les solutions linguistiques que la ville apporte à la communication sociale ont toutes les chances de s'imposer à l'ensemble du pays : telle une pompe, la ville aspire du plurilinguisme et recrache du monolinguisme, et elle joue un rôle fondamental dans l'avenir linguistique de la région ou de l'État. (Voix)

Moussirou-Mouyama indique que « $30 \%$ des enfants de couples linguistiquement mixtes ont le français pour langue première » en zone urbaine en 2000 (49). Ce chiffre a augmenté puisque Diane Bagouendi-Bagère Bonnot avance celui de 41\% en 2007, soit plus de 300000 habitants (65). Nous pensons comme Calvet que « la domination par les locuteurs de cette (ou de ces) langue(s) urbaine(s) est l'un des signes de l'intégration à la ville, en même temps que le choix collectif de cette (ces) langue(s) préfigure l'avenir linguistique du pays. (...) La ville apparaît comme un facteur d'unification linguistique » (135-136). L'urbanisation transforme la société en ce qu'elle change le statut des langues en présence. La langue véhiculaire, en l'occurrence le français, devient un instrument de communication référentielle, symbole de neutralité, d'objectivité, d'intégration à la ville et d'unification du pays.

\subsection{Français langue vernaculaire}

L'expansion du français a abouti à une situation de vernacularisation puisqu'il s'est introduit à l'intérieur des foyers et est devenu une langue domestique (= dévéhicularisation) et une langue première. «Le processus de vernacularisation présente un caractère identitaire de la langue qui prend alors le pas sur celui de neutralité, par la spécificité de son usage et sa constitution en une variété discernable et reconnue » (Manessy, «Vernacularité », 413).

Le français vernacularisé se présente comme une variété de français qui constitue une norme endogène. Ce français est issu de variétés distinctes de la norme exogène qui ont évolué selon une dynamique interne jusqu'à produire une variété qui présente des traits d'autonomie relative par rapport au français standard. L'usage courant de cette variété est admis par l'ensemble des locuteurs comme ordinaire, neutre et appartenant à la majorité de la communauté.

\section{Le français vernacularisé}

La vernacularisation entraîne une «simplification des structures grammaticales et l'élaboration compensatoire des moyens d'expression» (Manessy, Créoles, 96) par «un appareil morphologique surabondant dont chaque locuteur se sert avec une exactitude qui est à la mesure de sa capacité à imiter la norme » (97) endogène. La variation s'exprime dans un premier temps à travers la création lexicale puis, dans un second temps, dans la structure 
grammaticale de la langue avec notamment le double marquage des personnes sujets P3 et P6 et les changements dans le discours rapporté.

\subsection{La création lexicale}

La variation apparaît dans le lexique, premier témoin de la vernacularisation. En 2000, Suzanne Lafage et Karine Boucher, dans une étude sur la variation du français parlé au Gabon, ont élaboré un inventaire non exhaustif du lexique ayant subi la variation ; lexique recueilli lors d'enregistrements de français parlé, dans la rue, les émissions télévisées ou radiodiffusées, sur le web, dans la presse, la littérature.

La création lexicale propose par exemple comme expression avoir le carreau, qui signifie en argot avoir du succès (92); ou de verbes comme bober, vb. intr., de l'argot bobard, mentir (60), bastiller, vb. tr., qui signifie rouer de coups, battre, dérivé du mot bastille, qui désignait à l'origine la prison parisienne et qui au Gabon signifie les coups, certainement ceux administrés aux prisonniers, du moins dans l'imaginaire gabonais (49); ou gréver, vb. intr., faire la grève (204). Les verbes proposés sont des infinitifs en /-e/. La terminaison /-e/ semble être le suffixe privilégié pour créer des verbes. Cela se justifie par le fait que leur conjugaison est plus aisée à manier que celle des «autres verbes». Il y a moins d'irrégularités et de radicaux à maîtriser. La langue va au plus simple et la néologie verbale suit le principe de l'alignement sur la conjugaison des verbes en /-e/. Toutes les variétés de français tendent à choisir un radical verbal unique et des terminaisons aisées à utiliser, propres à ces verbes. Bien que les formes soient non standard en français de France, la création lexicale, comme il a été démontré, est régie par des règles définies dans les grammaires traditionnelles. Elle est rarement le fruit du hasard. Certains mots subissent des restrictions de sens comme échouer (159), qui signifie rater un examen, ou préparer, devenu intr., faire la cuisine, préparer le repas (331), depuis, employé seul signifie de longue date, depuis longtemps (142), et jus désigne un soda (228).

\subsection{Double marquage des personnes sujets P3 et P6}

L'analyse linguistique se fonde sur des corpus de français parlé au Gabon. Les locuteurs, au nombre de huit, sont tous des Gabonais originaires de la ville d'Oyem, au nord du pays, et possèdent la même langue maternelle, la langue ethnique le fang. Le français apparaît donc comme leur langue seconde. Le seul paramètre qui les différencie est le critère de l'âge. Quatre d'entre eux sont de jeunes adultes, les quatre autres, des personnes âgées. L'âge induit une différence de niveau de scolarisation : les plus jeunes ont tous le BEPC et les plus âgés ont été peu ou non scolarisés. Par conséquent, chaque groupe de locuteurs possède son propre mode d'appropriation de la langue française. En effet, les jeunes adultes 
par le biais de l'école ont été confrontés à la norme du français alors que les personnes âgées ont acquis le français 'sur le tas' par imitation de ceux qui le pratiquaient. Les corpus oraux ont été élaborés dans le cadre d'une thèse (Italia) qui porte sur l'analyse linguistique du verbe dans la langue française employée à l'oral par différents groupes de locuteurs gabonais. Le verbe subissant de profondes modifications formelles dans les corpus des locuteurs peu ou non scolarisés est transcrit en phonétique afin d'éviter toute interprétation arbitraire et hasardeuse.

D'un point de vue grammatical, nous observons dans le français parlé par des locuteurs peu ou non scolarisés un phénomène récurrent, celui de la présence quasi systématique du pronom personnel sujet P3 ou P6 en plus de la séquence lexicale.

Ex : les enfants [ipar] en brousse - pour-euh [HerHe] le - quelques bois [taje]

La neutralisation du pronom [il] s'opère par l'absence de la marque de la pluralité dans la terminaison verbale.

Nous ne sommes pas en présence d'une relation anaphorique du double marquage puisque celle-ci doit se situer à trois niveaux : l'indication du rang de la personne ; du nombre singulier ou pluriel ; du genre masculin ou féminin pour les P3 et P6. Pour les locuteurs peu ou non scolarisés, le pronom $[i l]$ perd la marque du nombre mais maintient l'indication du rang de la personne, P3 ou P6.

Le genre apparaît problématique car $[i l]$ peut reprendre une séquence lexicale au féminin.

Ex : ma tante [il vjC] de [murir] à Bitam

Occurrence en concurrence avec le pronom personnel sujet féminin elle :

Ex : une femme - à l'âge de trente quarante ans - elle [na] jamais [dOne] l'enfant depuis sa naissance

A travers la neutralisation du pronom clitique, la relation apparaît purement syntaxique. «Les pronoms clitiques fonctionneraient tels des affixes du verbe pour marquer la fonction syntaxique des syntagmes nominaux disloqués qui ne seraient qu'une possibilité de lexicalisation»(Blasco-Dulbecco 52). Mylène Blasco-Dulbecco indique «l'orientation pragmatique de la dislocation (...) [puisque] les deux éléments coréférentiels de la dislocation ont des fonctions syntaxiques différentes et complémentaires » (55).

\subsection{Le discours rapporté}

D'un point de vue syntaxique, dans le discours rapporté, la variation bouscule le mode d'ancrage du discours indirect (DI) à la fois dans les corpus des locuteurs peu ou non scolarisés et dans ceux des locuteurs ayant le BEPC. 
La dépendance syntaxique du discours cité (DCé) au discours citant (DCt) est conditionnée par le subordonnant que. Katja Ploog (304) le définit ainsi : «doté d'une charge sémantique très faible et ayant pour unique fonction de créer la connexion entre deux séquences propositionnelles, que est le terme conjonctif par excellence ». Ce morphème grammatical, réduit à sa fonction syntaxique, induit par sa présence celle du DI. La variation se manifeste par l'absence de la réalisation de la dépendance syntaxique malgré la présence de que.L'occurrence suivante montre l'absence de concordance des temps malgré la présence de que:

Ex : mon père [mavedi] - M. M. - que il [fomarje] beaucoup des femmes

Le verbe du DCé $[f o]$ aurait dû être à l'imparfait, temps conditionné par la dépendance syntaxique établie par que et par le verbe recteur au passé [mavedi]. L'absence de concordance des temps brouille la démarcation entre le DI et le DD (discours direct) puisque le temps employé dans le DCé ([fo]: forme du présent) serait celui utilisé si le DCé avait été un DD.

Il n'opère pas non plus de changement syntaxique :

Ex : le petit Hans - il me disait que non - - toi - voyageur qui n'as pas de chemin construis ton avenir en travaillant

La séquence jussive du DD construis deviendrait en DI un infinitif introduit par la préposition $d e$, sans la conjonction que puisque la subordonnée infinitive ne se construit pas avec ce subordonnant.

La réflexion pousse à s'interroger sur le statut du DCé et sur le rôle syntaxique opéré réellement par que en raison de la rareté des transpositions caractéristiques du DI du français standard. Le DCé semble se situer entre le DI et le DD dans son fonctionnement syntaxique. Quant à que, Ploog (305) s'interroge également sur son rôle : «il n'est pas établi qu'il s'agisse d'une subordination car il parait difficile d'identifier des contraintes relatives au contexte d'enchâssement. Plus exactement, la notion de subordination réfère à une réalité linguistique trop restrictive pour rendre compte de l'élaboration syntaxique observée, trop variable pour s'exprimer en termes de contraintes ».

Aucun des phénomènes indiqués ne se rencontrent dans le français décrit dans les grammaires et les dictionnaires du français de France. En revanche, même si la variation crée des néologies non recensées en France, et est donc transgressive vis à vis de ce même français décrit dans les grammaires et les dictionnaires, elle suit la création verbale selon des règles de ce même français. Il y a peut-être transgression mais il y a également un respect de la création selon la norme prescriptive. 
Pour les exemples de variation liés à la grammaire, il y a un non-respect des règles établies. La neutralisation du pronom clitique relève d'un sociolecte (les peu ou non scolarisés) alors que les changements d'insertion du DR se rencontrent dans le français parlé de tous les locuteurs gabonais, quel que soit leur niveau d'étude. Nous remarquons que ces présupposées transgressions n'aboutissent pas à une absence d'organisation du discours. Au contraire, nous voyons émerger une autre organisation des énoncés, pas encore stabilisée mais laissant apparaître des tendances syntaxiques, qui seront peut-être la norme de demain si elles sont reconnues officiellement comme telles par les Autorités gabonaises.

Nous parlons de transgressions mais est-ce bien pertinent de nommer ces phénomènes linguistiques comme telles alors que la population gabonaise ne maîtrise pas la norme prescrite, condition indispensable à ces mêmes transgressions ?

\section{Progression du français}

La progression du français s'est établie par son appropriation en tant que langue identitaire par le biais de l'oralité.

\subsection{Français langue identitaire}

D'un point de vue sociolinguistique, la langue est le reflet de l'identité sociale et historique, de la communauté qui la pratique. Que représente le français ? L'identité de la communauté française. Y a-t-il la possibilité de transposer totalement l'identité française sur l'identité gabonaise ? Cela reviendrait à supposer que la société gabonaise est identique à celle de la France. L'histoire et la sociologie ont prouvé que non. Les locuteurs au Gabon peuvent-ils alors se reconnaitre dans le français prescriptif ?

Nous avons vu l'évolution de la société gabonaise par l'urbanisation et le besoin de recourir à une langue identitaire à l'échelon national. L'évolution d'une société entraine obligatoirement des modifications linguistiques afin que la langue se réajuste à la société qui l'utilise. Le français du Gabon n'est ni le reflet d'une norme figée et lointaine ni celui d'une culture qui lui est étrangère.

Par comparaison, le français de France a créé des néologismes liés aux nouvelles technologies comme tablette tactile, le verbe twitter, ou liés au monde professionnel comme le mot manager, de l'anglais to manage (mener, diriger une affaire). Le français connaît des modifications syntaxiques comme après que + subj. au lieu de l'indicatif et malgré que bien que fautif commence à apparaître dans les grammaires où il est indiqué que son usage est toléré. Tout évolue parce que la société évolue, change, se transforme. La langue n'est pas 
figée, elle est dynamique donc elle évolue également en parallèle de la société pour continuer à être la représentation identitaire de cette même société.

Bien que ce soit le même pays, parlons-nous la langue de Chrétien de Troyes, de François Villon, de Montaigne ou de Louis XIV ? Avons-nous, nous locuteurs de français du vingt-et-unième siècle, l'impression de parler un français transgressif? Il ne peut être transgressif par rapport à l'ancien français ou le français classique puisque ces français correspondaient chacun à une société aujourd'hui révolue, à savoir la société du Moyen-Age et celle de l'ancien régime. Notre société actuelle, laïque et républicaine, ne peut donc s'identifier à celles précédemment citées. Puisque les sociétés sont différentes, la langue pratiquée par chacune d'elle l'est automatiquement.

La transgression apparaît donc comme la condition nécessaire à la survie de la langue. Elle est indispensable pour qu'une langue continue d'exister en tant que reflet d'une société en perpétuelle mutation. Elle participe au progrès et à l'évolution d'une communauté.

\subsection{L'oralité}

Une dimension importante doit être prise en considération dans l'étude de la variation du français en Afrique noire francophone : le contexte oral. En effet, le français est avant tout une langue orale car la ou les variétés qui évoluent sur les territoires africains relèvent toutes de l'oralité. Cela a une implication dans la variété du français parlé vernacularisé car le contexte de l'oral, quel que soit le locuteur, se distingue par une absence de pression normative ayant pour conséquence une restructuration du système allant vers la suppression des règles perçues comme redondantes. La situation d'oralité implique donc une certaine organisation du discours et de son contenu (Moseng Knutsen 30).

L'erreur qui est souvent commise dans l'analyse de ces variétés de français est de les étudier par le prisme de l'écrit. Cela apparaît donc comme paradoxal. De plus, la langue orale accumule les préjugées contre elle car elle est réputée fautive, populaire et vulgaire.

Deux contradictions ont été soulevées : celle où l'on juge une langue orale qui possède ses propres spécificités par le prisme de la norme prescriptive, c'est-à-dire de la langue écrite. L'autre, que nous avons vue, sous-entend que pour qu'il y ait transgression, il doit y avoir au préalable connaissance de règles préétablies mais il a été démontré que la population au Gabon ne connaît ni ne maîtrise la norme prescriptive, ou norme fantasmée. Donc comment peut-il y avoir transgression de règles alors qu'elles sont inconnues par cette même population?

Dans cette perspective, la transgression ne s'envisage plus comme le simple fait de contrevenir à une règle, ou comme un écart, mais comme une dimension constructive et 
linguistique par l'adaptation au contexte local. Cette autonomisation va se manifester en profondeur par l'apparition de phénomènes sous-jacents et récurrents comme la réorganisation des champs sémantiques, les déplacements et dérèglements de sens, en un mot par tous les phénomènes liés à la production du sens en discours et qui font du français d'Afrique un véritable domaine de création identitaire.

\subsection{Critères d'acceptabilité d'une variété}

« La création d'un autre usage permet de s'assumer, d'exister » (Dumont et Maurer 179) ; c'est-à-dire d'avoir sa propre réalité et d'être autonome. Pierre Dumont et Bruno Maurer proposent trois critères d'acceptabilité de la variation par l'usage. Le premier critère concerne l'utilité du mot; son utilité référentielle doit désigner une réalité absente de la langue source. «Il va de soi qu'un nouvel environnement culturel et naturel puisse susciter de nouveaux besoins communicatifs » (180). L'utilité expressive relève de modèles rhétoriques particuliers. Par les innovations linguistiques, le français ne se réduit pas à un rôle d' «instrument d'étiquetage, parfaitement impersonnel ». Le second critère concerne les différents procédés néologiques qui doivent se conformer à ceux du français prescriptif. Le troisième, la vitalité lexicale se signale par la fréquence d'emploi et par la productivité d'un mot « en tant que base de dérivation, de composition, de formation de syntagmes et par sa richesse sémantique »(182). Nous rajoutons comme critères, la diffusion diatopique (lieu), diastratique (classe sociale) et la légitimation d'une variété par sa valeur identitaire. Ces critères sont objectifs car théoriquement quantifiables, plus les unités linguistiques seront récurrentes, plus elles seront considérées comme appartenant à l'usage collectif. Les écarts ne peuvent plus être considérés comme tels puisqu'ils sont admis par toute une collectivité linguistique qui se reconnaît dans cet usage.

Le locuteur doit légitimer l'usage des unités linguistiques qui donne à la langue, considérée comme une construction sociale, sa cohésion. Le lexique « doit porter les marques de l'appropriation du locuteur: en Afrique, plus qu'ailleurs, c'est en pratiquant un réglage endogène du sens, à la fois en langue et en discours que le non-natif fait de la langue française un lieu de production du sens, de représentation du réel, de catégorisation de l'expérience singulière. Sa propriété » (186). La consolidation d'une norme endogène passe donc par un double mécanisme d'appropriation de la langue, d'une part, à travers une affirmation toujours plus nette de la valeur et de la légitimité de certains marqueurs identitaires, et, d'autre part, à travers la prise de conscience du droit des locuteurs à fixer leurs propres normes linguistiques. La langue évolue vers une autonomie et une reconnaissance sociale et linguistique. La variété populaire devient le bon usage et le reflet 
d'une société et d'une histoire collective. Elle symbolise l'instrument de la cohésion nationale cimentée par son autonomisation ; cohésion qui entraine sa cohérence.

La transgression ne le devient que quand elle est considérée comme telle par l'interlocuteur. Elle entraine un jugement négatif et des à priori sur le locuteur qui l'emploie. Ce présupposé pose un problème dans l'appréciation des faits de langue liés à cette «transgression». Nous avons vu que dans le cas du Gabon, ce qui pourrait être nommé abusivement transgression participe en fait à l'évolution de la langue, à son autonomisation et à sa survie, éléments qui n'endossent pas les mêmes connotations puisqu'elles sont positives. Par le prisme de la variation et de l'appropriation, cette transgression devient un processus généralisé et linguistique d'une construction identitaire et sociale. Il s'agit donc d'une progression, d'un développement continu du français parlé au Gabon.

\section{Conclusion}

Le français efface les inégalités sociales, gomme les défaillances linguistiques dans la langue ethnique et fait oublier l'appartenance ethnique. Il met tous les locuteurs, gabonais ou non, sur le même pied d'égalité» (Bagouendi-Bagère Bonnot 51). Ce qui permet alors l'émergence d'un sentiment de cohésion et d'appartenance nationale. Le français apparaît comme une véritable langue de partage.

Par le prisme de l'appropriation et de la variation, le français subit des modifications linguistiques avec des tendances qui s'affirment et qui ne paraissent en rien marginales car tous les locuteurs les jugent «normatives ». Grâce à ces progressions, le français s'affiche comme une langue identitaire de la société gabonaise, et surtout comme une langue vivante, c'est-à-dire une langue en perpétuel mouvement où la progression participe à son évolution et à son maintien en vie par l'adéquation entre sa pratique, sa représentation et son identité nationale.

La limite normative s'efface au profit de la créativité. Cette variété de français est dans un processus d'autonomisation comme les créoles par rapport au français ou le français par rapport au latin. Les tendances d'aujourd'hui seront la norme de demain. Rester dans l'optique «transgression », c'est s'empêcher d'apprécier la créativité linguistique pour ce qu'elle est, et, le non franchissement de présupposés figés en est un frein. La transgression, finalement, signifie progression et appropriation, des qualifications positives. 


\section{Bibliographie}

Baggioni, Daniel. «Norme», in Sociolinguistique Concepts de base. Dir Marie-Louise Moreau. Liège : Mardaga, 1997. 217-218.

Bagouendi-Bagère, Diane. «Etudes des procédés de néologie lexicale dans le français parlé au Gabon ». Mémoire de D.E.A. sous la direction de A. Queffélec, U de ProvenceAix-Marseille I, 1999.

Bagouendi-Bagère Bonnot, Diane. «Le français au Gabon: représentations et usages ». Thèse de doctorat, U de Provence, 2007.

Blasco-Dulbeco, Mylène. Les dislocations en français contemporain - Études syntaxiques. Paris : Champion, 1999.

Boucher, Karine et Suzanne Lafage. Le lexique français du Gabon (Entre tradition et modernité). Le français en Afrique 14. Nice: Institut de linguistique française CNRS, 2000.

Bronckart, Jean-Paul. «Fonctionnement langagier et entreprises normatives ». Dir. G. Schoeni et al. La langue française est-elle gouvernable? Neuchâtel / Paris: Delachaux et Niestlé, 1988. 109-131.

Calvet, Louis-Jean. «Français et urbanisation». Le français dans l'espace francophone. Description linguistique et sociolinguistique de la francophonie. Tome 1, Paris: Champion, coll. Politique linguistique, 1993.431-447.

---. Les voix de la ville, Introduction à la sociolinguistique urbaine. Paris : Payot, coll. Essais, 1994.

---. «Véhiculaire ». Sociolinguistique Concepts de base. Dir. Marie-Louise Moreau. Liège : Mardaga, 1997. 289-290.

---. La guerre des langues et les politiques linguistiques. Paris : Hachette Littérature, coll. Pluriel, 1999.

---. «La ville et la gestion in vivo des situations linguistiques ». Le plurilinguisme urbain Actes du colloque de Libreville Les villes plurilingues 25-29 septembre 2000. Dir. Louis-Jean Calvet et Auguste Moussirou-Mouyama. Agence intergouvernementale de la francophonie, Institut de la francophonie / Paris : Didier Érudition, coll. Langues et développement, 2000. 11-30.

Chaudenson, Robert. La créolisation : théorie, applications, implications. Paris : Harmattan, Institut de la francophonie, coll. Langues et développement, 2003. 
---, Raymond Mougeon et Edouard Beniak. Vers une approche panlectale de la variation du français. Paris: Harmattan, Institut de la francophonie, coll. Langues et développement, 1993.

Dumont, Pierre et Bruno Maurer. Sociolinguistique du français en Afrique francophone Gestion d'un héritage, devenir d'une science. Vanves : EDICEF, U francophones, AUPELF-UREF, 1995.

Gadet, Françoise. La variation sociale en français. Paris : Ophrys, coll. Français l'essentiel, 2003.

Italia, Magali. «Variation et variétés morphosyntaxiques du français parlé au Gabon ». U de Provence. Thèse de doctorat, 2011.

Manessy, Gabriel. «Vernacularité, vernacularisation». Le français dans l'espace francophone. Description linguistique et sociolinguistique de la francophonie. Tome 1. Paris : Champion, coll. Politique linguistique, 1993, pp.407-417.

---. Le français en Afrique noire - Mythes, stratégies, pratiques. Paris : Harmattan, coll. Espaces francophones, 1994.

---. Créoles, pidgins, variétés véhiculaires - Procès et genèse. Paris : CNRS, coll. Sciences du langage, 1995.

Moreau, Marie-Louise, dir. Sociolinguistique Concepts de base. Liège : Mardaga, $1997 .$.

Mougeon, Raymond et Edouard Beniak. «Le non-accord en nombre entre sujet et verbe en français ontarien : un cas de simplification ? » Présence Francophone 46 (1995) : 53 65.

Moseng Knutsen, Anne. «Variation du français à Abidjan (Côte d'Ivoire) Etude d'un continuum linguistique et social ». U d'Oslo, Norvège. Thèse de doctorat, 2007.

Moussirou-Mouyama, Auguste. «Libreville, ancien village d'esclaves libérés: des contraintes de la langue à la liberté des citoyens ». Le plurilinguisme urbain - Actes du colloque de Libreville Les villes plurilingues 25-29 septembre 2000. Dir. LouisJean Calvet et Auguste Moussirou-Mouyama. Agence intergouvernementale de la francophonie, Institut de la francophonie / Paris : Didier Érudition, coll. Langues et développement, 2000. 31-51.

--- et Thierry de Samie. «La situation sociolinguistique du Gabon». Le français dans l'espace francophone. Description linguistique et sociolinguistique de la francophonie. Tome 2. Paris : Champion, coll. Politique linguistique, 1993. 603-613. 
Ploog, Katja. «Stratégies et structures du discours rapporté dans les récits abidjanais ». Le discours rapporté dans tous ses états, Actes du Colloque International Bruxelles, 8-11 novembre 2001. Paris : Harmattan, coll. Sémantiques, 2004. 297-306.

Simo Nguemkam-Souop, Adeline Larissa. «La variation du français au Cameroun Approche sociolinguistique et syntaxique ». U de Provence. Thèse de doctorat, 2009.

\section{NOTE}

${ }^{1}$ Nous entendons par plurilinguisme, « les diverses langues au sein d'un ensemble national donc un Etat » selon la définition de Robert Chaudenson (Créolisation, 25). 